\title{
Effectiveness of Post-Discharge Case Management in General- Medical Outpatients: A Randomized, Controlled Trial
}

\author{
Corine H.M. Latour, C.N.S., R.N., Rien de Vos, Ph.D. \\ Frits J. Huyse, M.D., Ph.D., Peter de Jonge, Ph.D. \\ Liesbeth A.M. van Gemert, M.Sc., R.N., Wim A.B. Stalman, Prof., M.D., Ph.D.
}

This study was initiated to determine the impact of post-discharge, nurse-led, home-based case management intervention on the number of emergency readmissions, level of care utilization, quality of life, and psychological functioning. Patients discharged home from a general hospital $(N=147)$ were randomly assigned to usual care or nurse-led, home-based, case management intervention. During the 24 weeks of follow-up, no difference between the two groups was found for readmission, care utilization, quality of life, or psychological functioning. Patients in the control group tended to move sooner to non-independent living accommodation than patients in the nurse-led, home-based, case management intervention group.

(Psychosomatics 2006; 47:421-429)

$\mathrm{H}$ ospitals are confronted with an increasing number of vulnerable patients, such as elderly patients, and those with chronic diseases, psychiatric comorbidity, and a limited social network. In recent years, nurse-led disease management has been found to be an effective strategy for dealing with vulnerable patients with specific diseases (e.g., congestive heart failure or diabetes mellitus). ${ }^{1-5}$ Whereas disease management focuses on specific illnesses, case management is concerned with an optimization of multidisciplinary treatment without being focused on only one specific illness. The effectiveness of case management has mainly been studied in elderly patients. ${ }^{6-8}$ It is still unclear to what extent care-coordination based on case management is an effective strategy for dealing with multi-morbid complaints. ${ }^{9}$ We do know that the INTERMED is a reliable and valid instrument to identify patients in need of case management, ${ }^{10-12}$ and we have already demonstrated, in an earlier study for patients in a general hospital, that care designed according to the INTERMED method resulted in better discharge status and a reduction in the duration of hospitalization. ${ }^{13,14}$ However, it is still unclear the extent to which case management is an effective strategy for dealing with a post-discharge outpatient population with multimorbid complaints.
The objective of this study is to determine the impact of post-discharge, nurse-led, home-based, case management intervention (NHI) on the number of emergency readmissions, the level of care utilization, quality of life, and psychological functioning.

\section{METHOD}

Design, Randomization, Blinding, and Location

To assess the impact of the case management intervention relative to usual care, we conducted a randomized, controlled trial in patients discharged from a hospital. The

Received July 20, 2005; revised December 6, 2005; accepted December 15, 2005. From the Psychiatric Consultation and Liaison Service, VU University Medical Center, Amsterdam; the Dept. of Clinical Epidemiology and Biostatistics, Academic Medical Center, Univ. of Amsterdam; the Dept. of General-Internal Medicine, Groningen Univ. Hospital; the Dept. of Psychiatry, Univ. of Groningen; and the Dept. of General Practice, Institute for Research in Extramural Medicine, VU Univ. Medical Center, Amsterdam, The Netherlands. Address correspondence and reprint requests to C.H.M. Latour, Psychiatric Consultation and Liaison Service / Rm. 2 D 22, VU University Medical Center, P.O. Box 7057 1007, MB Amsterdam, the Netherlands. e-mail: chm.latour@vumc.nl (C) 2006 The Academy of Psychosomatic Medicine 
patients were allocated either to the case management intervention or care-as-usual, and were compared on emergency readmissions, care utilization, quality of life, and psychological functioning. The study was carried out in the Netherlands, at the VU University Medical Center in Amsterdam, between October 2001 and December 2003.

Randomization took place on discharge by an independent co-worker using a concealed randomization list stratified according to age ( $<60,60-69, \geq 70$ years). Due to the nature of the intervention, blinding of the patients was not possible. The outcome measurements of emergency readmission and care utilization were objectively determined by analyzing research forms and medical records, and quality of life and psychological functioning were assessed by means of patient self-report.

\section{Participants}

Included were patients admitted to the departments of internal medicine, gastroenterology, pulmonology, and cardiology, who had been admitted at least once ( $\geq 2$ nights) in the previous 5 years since the current admission. They had to be a resident in the municipality of Amsterdam, age 18 years or older, and able to speak Dutch or English (or have a relative who spoke Dutch or English). Excluded were patients who had been discharged to non-independent living accommodations, patients who had a Mini-Mental State Exam (MMSE) ${ }^{15}$ score $<21$ (and had no relative who could help with completing the questionnaires), or with planned readmissions (e.g., chemotherapy). All patients were informed about the background and procedures of the trial (orally and in writing) and had to give informed consent. The Medical Ethical Committee of the VU University Medical Center approved the research protocol.

\section{Intervention}

Within 3-10 working days after hospital discharge, a case manager (trained nurse-specialist), visited the patient at home. The complexity of the patient's status was assessed by means of the INTERMED, which consists of 20 items, each measured on a 4-point scale (0-3), with a total score ranging from $0-60$. A score of $\geq 21$ points indicates the need for case management. ${ }^{14}$ In order to identify factors associated with case complexity, the case manager and the medical supervisor used the INTERMED vignette to assess patients with specific care needs on six factors: chronic physical illness, psychological vulnerability, diagnostic complexity, compliance/adherence, physical dependency, and social functioning, which indicated the required care. ${ }^{10,11,13,14}$ Activities of daily living (ADLs) and instrumental activities of daily living (IADLs) were assessed by means of questionnaires. ${ }^{16}$ After consulting the medical supervisor, the case manager discussed the INTERMED care plan with the patient, the general practitioner, and other people who were involved in the patient's treatment.

For the care plan, the following interventions were considered: psychosocial support for the patient and relatives (e.g., structuring, supportive interventions); mediation between patient and medical specialists or allied health professionals and referral; and improvement of compliance with medication, physical exercises, diet, smoking, and alcohol recommendations. Self-management was promoted, as well as keeping appointments with care-providers. ${ }^{3}$ Depending on the care plan, the case manager made regular home visits (at least every 2 months), and the patients were contacted by telephone. The case-management intervention was tailored to the patients' needs, and, therefore, not all interventions were the same for all patients. After 24 weeks, the case-management intervention was ended, and the patient's general practitioner received a letter reporting on the case manager's findings.

\section{Usual Care}

Patients in the control group did not receive a casemanagement intervention after discharge from the hospital, but received usual care. Care in this group was provided according to the recommendation of the medical specialist and the general-practitioner.

\section{Data Collection and Outcome Measures}

The primary outcome was the number of emergency readmissions at 24 weeks. Readmissions and the time from discharge to the first emergency readmission were recorded on a checklist that was completed by the patient or a relative and cross-referenced with the hospital databases. Careutilization, as secondary outcome, was assessed by means of a specifically developed care diary that was filled in 3 times for a period of 4 weeks by the patient or a relative (1-4 weeks, 9-12 weeks, and 21-24 weeks). Information about medication was provided by the patient's pharmacist.

Quality of life was assessed at 0, 12, and 24 weeks with the SF-36 Health Survey. This questionnaire contains 36 items, which are combined to 8 subscales. The scores on each of the subscales range from 0 to 100: higher scores indicate better health status. ${ }^{17,18}$ Psychological functioning 
was assessed with the Hospital Anxiety and Depression Scale (HADS). This questionnaire contains 14 items, which are combined into 2 subscales (Depression and Anxiety), which range from 0 to 21 : higher scores indicate more complaints. ${ }^{19}$

If the mailed questionnaires were not returned, the researcher made a follow-up telephone call after 5 business days and, if necessary, again after 10 business days.

\section{Sample Size}

On the basis of data from a pilot study and a literature search, the risk of readmission within 6 months was estimated to be $50 \%$. The hypothesis was that if this percentage could be reduced to $25 \%$, a total of 130 patients ( 65 per condition) would be needed (alpha: 0.05; power: 0.80). Extra patients were sampled, taking drop-out after randomization into account.

\section{Statistical Analysis}

The analysis was carried out according to the intention-to-treat principle. Effects on emergency readmissions were expressed in relative risks (RRs) and their 95\% confidence intervals (CIs). Kaplan-Meier analysis and a logrank test were applied to estimate the difference between the two groups in time-to-first emergency readmission. For the secondary outcomes, mean differences and 95\% CIs between the two groups for care-utilization were calculated by an independent-sample $t$-test, or a Mann-Whitney $U$ test in case of skewed distribution. For quality of life and psychological functioning, we calculated median differences and $95 \%$ CIs between the two groups.

In a multivariate analysis using linear-regression models, the effects estimated in univariate analysis were corrected for differences in baseline characteristics. A $p$ value $<0.05$ was considered to be statistically significant. The data were analyzed by SPSS for Windows, Version 11.0.

\section{RESULTS}

\section{Patient Characteristics}

Of the 1,219 eligible patients, 23 were discharged after office hours and could not be traced; 945 did not meet the inclusion criteria; and $43(3.5 \%)$ were unwilling to participate (Figure 1). Reasons given for non-participation were the following: patients felt too ill to fill in questionnaires (48.9\%; $\mathrm{N}=21)$; did not want home visits $(11.6 \%$;
$\mathrm{N}=5$ ); did not want to be reminded of the hospital (11.6\%; $\mathrm{N}=5)$; objected to the research aims $(11.6 \% ; \mathrm{N}=5)$; or had other reasons $(16.3 \% ; \mathrm{N}=7)$. A total of 208 patients were randomly assigned to a treatment condition between October 2001 and December 2003: 107 to the control group and 101 to the case-management intervention. There were 61 dropouts $(29.3 \% ; 38$ in the control group and 23 in the case-management intervention group). Reasons given for dropout were withdrawal of consent because of feeling too ill or that patients did not want home visits after all $(65.6 \% ; \mathrm{N}=40) ; 15$ patients $(24.6 \%)$ died, and $6(9.8 \%)$ were lost to follow-up. A total of 147 patients $(70.7 \%)$ completed the study with a follow-up of 24 weeks. These patients represented a population with a mean age of 64 years (standard deviation [SD]: 6.6), half of whom were men (Table 1). The majority of the patients lived with a partner, and $37 \%(\mathrm{~N}=54)$ lived alone. Most of the patients $(81.6 \%)$ did not have a paid job or were retired. The mean duration of hospitalization was 11.6 days (SD: 12.2). The majority of patients had been admitted to the department of internal medicine $(41 \% ; \mathrm{N}=60)$, and $30.6 \%$ of the patients suffered from circulation problems according to ICD-9. Four patients were cognitively impaired (MMSE < 21). There were no statistically significant differences in baseline characteristics between the control group and the case-management intervention group, except for psycho-

\section{FIGURE 1. Patient Flow}

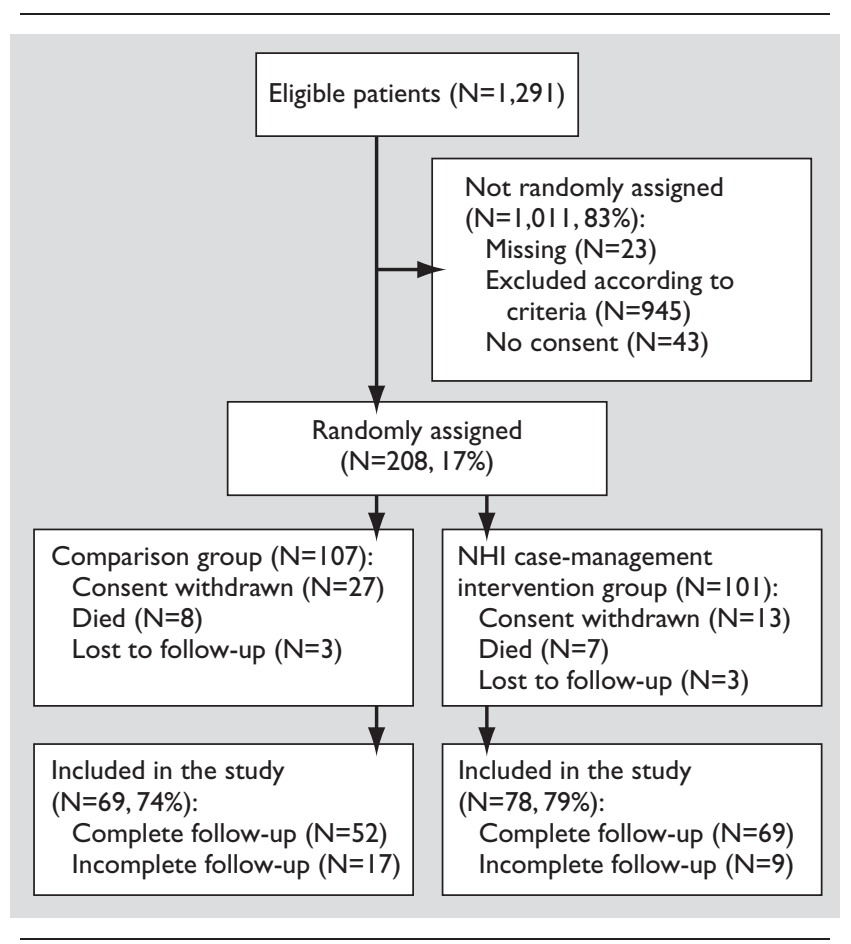


logical functioning (HADS score). With regard to quality of life (SF-36), the control group tended to have better mental health status and less pain (Table 1). Compared with those who completed the follow-up (control group, $\mathrm{N}=52$; case-management intervention group, $\mathrm{N}=69$ ) the baseline characteristics of the patients who did not complete the follow-up (control group, $\mathrm{N}=17$; case-management inter- vention group, $\mathrm{N}=9$ ) were not significantly different. Among the patients who did not complete the follow-up, no significant differences were found between the control group and the case-management intervention group (see Appendix 1). Among the dropouts, no significant differences were found between the control group and the casemanagement intervention group (see Appendix 2).

\begin{tabular}{|c|c|c|c|}
\hline & $\begin{array}{c}\text { Control Group } \\
(\mathrm{N}=69)\end{array}$ & $\begin{array}{c}\text { Case-Management } \\
\text { Intervention Group }(\mathbf{N}=78)\end{array}$ & $\mathbf{p}$ \\
\hline Age, years, mean $(\mathrm{SD})$ & $62.32(17.50)$ & $65.29(15.74)$ & 0.35 \\
\hline \multicolumn{4}{|l|}{ Gender, N (\%) } \\
\hline Male & $36(52.2)$ & $39(50)$ & \multirow[t]{2}{*}{0.79} \\
\hline Female & $33(47.8)$ & $39(50)$ & \\
\hline \multicolumn{4}{|l|}{ Marital status, $\mathrm{N}(\%)$} \\
\hline Married/living together & $42(60.9)$ & $51(65.4)$ & \multirow[t]{2}{*}{0.5} \\
\hline Living alone & $27(39.1)$ & $27(34.6)$ & \\
\hline \multicolumn{4}{|l|}{ Work status, $\mathrm{N}(\%)$} \\
\hline Not working & $54(78.3)$ & $66(84.6)$ & \multirow[t]{2}{*}{0.32} \\
\hline Working & $15(21.7)$ & $12(15.4)$ & \\
\hline Hospital length of stay, mean (SD) & $13.3(15.99)$ & $10.1(7.04)$ & 0.42 \\
\hline \multicolumn{4}{|l|}{ Specialty, N (\%) } \\
\hline Internal medicine & $24(34.8)$ & $36(46.2)$ & \\
\hline Gastroenterology & $15(21.7)$ & $12(15.4)$ & \\
\hline Pulmonology & $9(13.0)$ & $10(12.8)$ & \\
\hline Cardiology & $21(30.4)$ & $20(25.6)$ & \\
\hline \multicolumn{4}{|l|}{ Medical diagnoses by ICD-9, N (\%) } \\
\hline Endocrine disorder & $5(7.2)$ & $5(6.4)$ & \\
\hline Circulatory disorder & $19(27.5)$ & $26(33.3)$ & \\
\hline Respiratory disorder & $11(15.9)$ & $14(17.9)$ & \\
\hline Gastrointestinal disorder & $16(23.2)$ & $14(17.9)$ & \\
\hline Infectious disease & $4(5.8)$ & $3(3.8)$ & \\
\hline Other & $14(20.3)$ & $16(20.5)$ & \\
\hline Number of admissions in last 5 years, mean (SD) & $2.58(2.58)$ & $1.99(1.37)$ & \multirow[t]{2}{*}{0.44} \\
\hline INTERMED score, mean (SD) & - & $20.35(6.73)$ & \\
\hline \multicolumn{4}{|l|}{ Mini-Mental State Exam, N (\%) } \\
\hline$<21$ & $1(1.4)$ & $3(3.8)$ & \multirow[t]{2}{*}{0.44} \\
\hline$\geq 21$ & $68(98.6)$ & $75(96.2)$ & \\
\hline SF-36, median $(25 \%-75 \%)$ & Control Group & NHI Group & \multirow[t]{2}{*}{$\mathrm{p}$} \\
\hline Domains of SD-36 & $\mathrm{N}=69^{\mathrm{a}}$ & $\mathrm{N}=78^{\mathrm{b}}$ & \\
\hline Physical Functioning & $32.5(11-64)$ & $30.0(10-65)$ & 0.93 \\
\hline Physical Role & $0.0(0-25)$ & $0(0-0)$ & 0.26 \\
\hline Emotional Role & $66.7(0-100)$ & $0.0(0-100)$ & 0.11 \\
\hline Social Functioning & $50.0(33-67)$ & $44.4(22-56)$ & 0.18 \\
\hline Mental Health & $72.0(56-88)$ & $64.0(48-80)$ & 0.08 \\
\hline Vitality & $45.0(30-60)$ & $35.0(25-55)$ & 0.11 \\
\hline Pain & $61.1(33-97)$ & $44.4(33-67)$ & 0.08 \\
\hline General Health & $41.0(28-55)$ & $35.0(25-54)$ & 0.30 \\
\hline Change in Health & $50.0(25-69)$ & $50.0(25-75)$ & 0.62 \\
\hline Physical Health Total & $144(96-198)$ & $121.7(83-171)$ & 0.10 \\
\hline Mental Health Total & $211(156-286)$ & $179(109-257)$ & 0.06 \\
\hline \multicolumn{3}{|c|}{ Hospital Anxiety and Depression Scale, median (25\%-75\%) } & $\mathrm{p}$ \\
\hline Total score & $10(4.3-16)$ & $13(6.5-20)$ & 0.03 \\
\hline Anxiety total score & $4(2-7.8)$ & $6(3.5-10)$ & 0.04 \\
\hline Depression total score & $4(1.3-9)$ & $7(2-11)$ & 0.12 \\
\hline
\end{tabular}


Nurse-Led, Home-Based Intervention (NHI) Characteristics

In the case-management intervention group, $72 \%$ $(\mathrm{N}=56$ of 78$)$ of the first home visits lasted between 30 and 60 minutes; $13 \%$ had a shorter duration, and $15 \%$ had a longer duration (Table 2). With regard to the second and subsequent visits to these patients, $52 \%(\mathrm{~N}=41$ of 79$)$ had a duration of 30-60 minutes; $45.5 \%$ had a shorter duration, and only $2.5 \%$ had a longer duration. The visits to the outpatient clinic had a duration of 1 minute-30 minutes (79\%; $\mathrm{N}=26$ of 33). The telephone was used frequently (270 times, with a mean duration of 5 minutes- 15 minutes), either for contacting patients (151 times), or for consulting care-providers (119 times). The letters that were written to the general-practitioner or the medical disciplines for each patient took 10 minutes-20 minutes per letter to write.

Since the case-management intervention was tailored to the needs of each individual patient, the interventions were not the same for all patients. The INTERMED factors associated with case-complexity indicated the required care. The ADL and IADL scores for each visit provided indications for effective referral to home-care organizations. Referrals were recommended 49 times (Table 3). Psychosocial support was provided most frequently (394 times), and education least frequently (14 times). The reason for this low level of education recommendation was that patients were encouraged to seek information themselves (e.g., promoting self-management, 69 times).

\section{Outcomes}

Eleven patients in the control group (15.9\%) and 16 patients in the case-management intervention group (20.6\%) were readmitted for an emergency. The crude RR

\begin{tabular}{lrc}
\hline TABLE 2. Intensity of Visits and & Other Communications \\
\hline Communication & N & Duration Range, minutes \\
\hline Total visits & 258 & $30-40$ \\
First home visit & 78 & \\
Subsequent home visits & 79 & \\
Ambulatory clinic visit & 33 & \\
Last home visit & 68 & $5-15$ \\
Total telephone contacts & 270 & \\
Care-provider & 119 & $10-20$ \\
Patient & 151 & \\
Total letters & 78 & \\
Medical disciplines & 8 & \\
General-practitioner & 69 & \\
Patient & 1 & \\
\end{tabular}

(1.30; 95\% CI: 0.64-2.58) for emergency readmission remained similar after adjustment for baseline differences. Survival analysis showed no significant difference between the two groups in time from discharge to the first emergency readmission $(p=0.48$; Figure 2$)$.

For both groups, there was a total of 33 emergency readmissions $(11+16$ first readmission and 6 multiple readmissions). The median duration of all emergency readmissions was 11 days (range: 4-59) for the control group and 10.5 (range: 2-68) days for the case-management intervention group, but this difference was not statistically significant (95\% CI: -13 to 6.0 days). With regard to careutilization by the patients who completed the follow-up (control group, $\mathrm{N}=52$; case-management intervention group, $N=69$ ) the mean differences in general care utilization were not statistically significant. The differences in mean use of primary-care services were minimal, but compared with the control group, there was a tendency among patients in the case-management intervention group to make more use of home-care facilities and less use of nonindependent living accommodations. With regard to quality of life, the median difference in score for the separate dimensions of quality of life (SF-36) and psychological functioning (HADS) indicated an outcome in favor of the control group (Figure 3). After correction in multivariate analysis for differences in baseline characteristics, no sig-

\begin{tabular}{|cc|}
\hline TABLE 3. Content of Interventions, NHI Group & $\begin{array}{c}\text { Number of } \\
\text { Interventions } \\
\text { (non-complex/complex) }\end{array}$ \\
\hline Referrals & $49(12 / 37)$ \\
Medical disciplines & $34(5 / 29)$ \\
Mental health care & $4(0 / 4)$ \\
Home-care & $7(3 / 4)$ \\
Living accommodations & $4(4 / 0)$ \\
Psychosocial support & $394(144 / 211)$ \\
Patient & $279(107 / 172)$ \\
Relative & $115(37 / 77)$ \\
Enhancement of compliance & 171 \\
Medication & $29(8 / 21)$ \\
Appointments & $35(10 / 25)$ \\
Physical exercise & $33(6 / 27)$ \\
Diet & $31(10 / 21)$ \\
Smoking & $21(6 / 15)$ \\
Alcohol & $20(0 / 20)$ \\
Other & $2(1 / 1)$ \\
Promotion of self-management & $69(17 / 42)$ \\
Education & $14(6 / 8)$ \\
\hline NHI: Nurse-Led Home-Based Care-Management Intervention \\
a Non-complex: INTERMED $<21 ;$ complex: INTERMED $\geq 21$. \\
\hline
\end{tabular}


nificant differences between the groups were found with regard to the outcome on these two dimensions.

\section{DISCUSSION}

We could not demonstrate that case-management intervention is an effective strategy for dealing with a vulnerable post-discharge outpatient population. The case-management intervention did not result in a reduction of emergency readmissions or a reduction of care-utilization or an improvement in quality of life and psychological functioning. The study did demonstrate a trend toward a possible shift in the direction of home-care facilities and away from non-independent-living accommodation.

Our case-management study is unique because we studied a general population after discharge from hospital. Other studies in this field of research can be categorized into studies on ambulant case management and studies on disease management. Ambulatory case-management studies focus mainly on nonhospitalized elderly patients with a variety of diseases, whereas disease-management studies focus only on patients with one specific illness. It has been demonstrated that disease management is effective, ${ }^{1,3}$ and one could be tempted to think that disease management is sufficient. However, it is evident that a considerable number of patients suffer from multiple morbidities, and, for these patients, a focus on isolated diseases is often not sufficient. Disease management relies on protocols, and it is difficult to capture complex patients in these protocols. The INTERMED method is a reliable and valid instrument to FIGURE 2. Time to Emergency Readmission After Hospital
Discharge, days

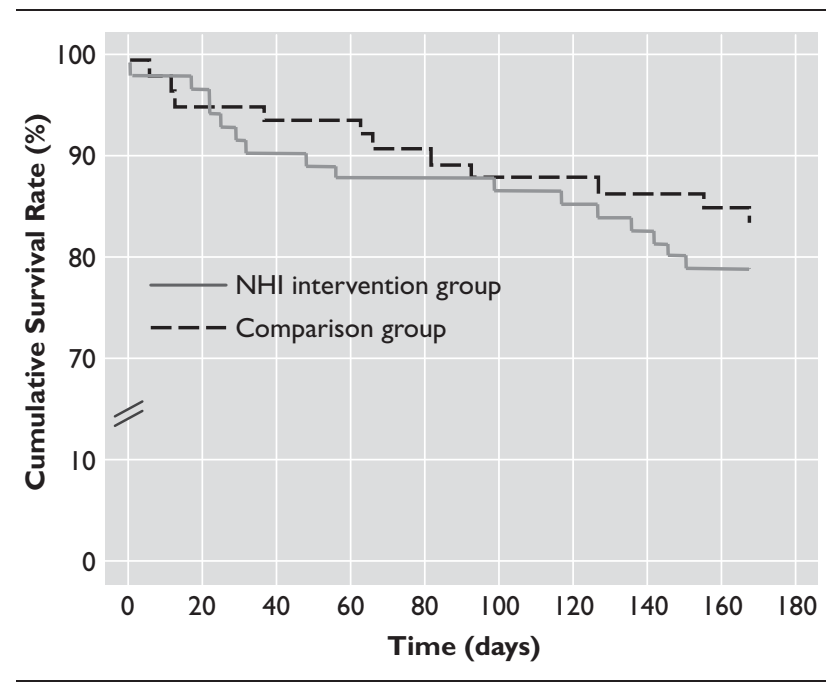

identify patients who are in need of case management, ${ }^{10-12}$ and it formulates the care for complex patients better than static protocols. We demonstrated in an earlier study that, for patients in a general hospital, care based on the INTERMED method resulted in better discharge status than usual care. ${ }^{13,14}$ However, we could not demonstrate in the present study that case-management is an effective postdischarge strategy, and we considered a number of methodological and clinical explanations for the above findings.

First, we considered the power of the study. We do not believe that the current findings result from a lack of power to demonstrate a smaller difference because, apart from the confidence intervals, the point estimates do not suggest a positive result.

Selective drop-out (Figure 1; incomplete follow-up), leaving more complex or severely ill patients in the case-

FIGURE 3. Case-Management: Median Differences in Quality of Life (SF-36) and HADS Anxiety, Depression and Total Scores Between the Control and the NHI Group After 24 Weeks

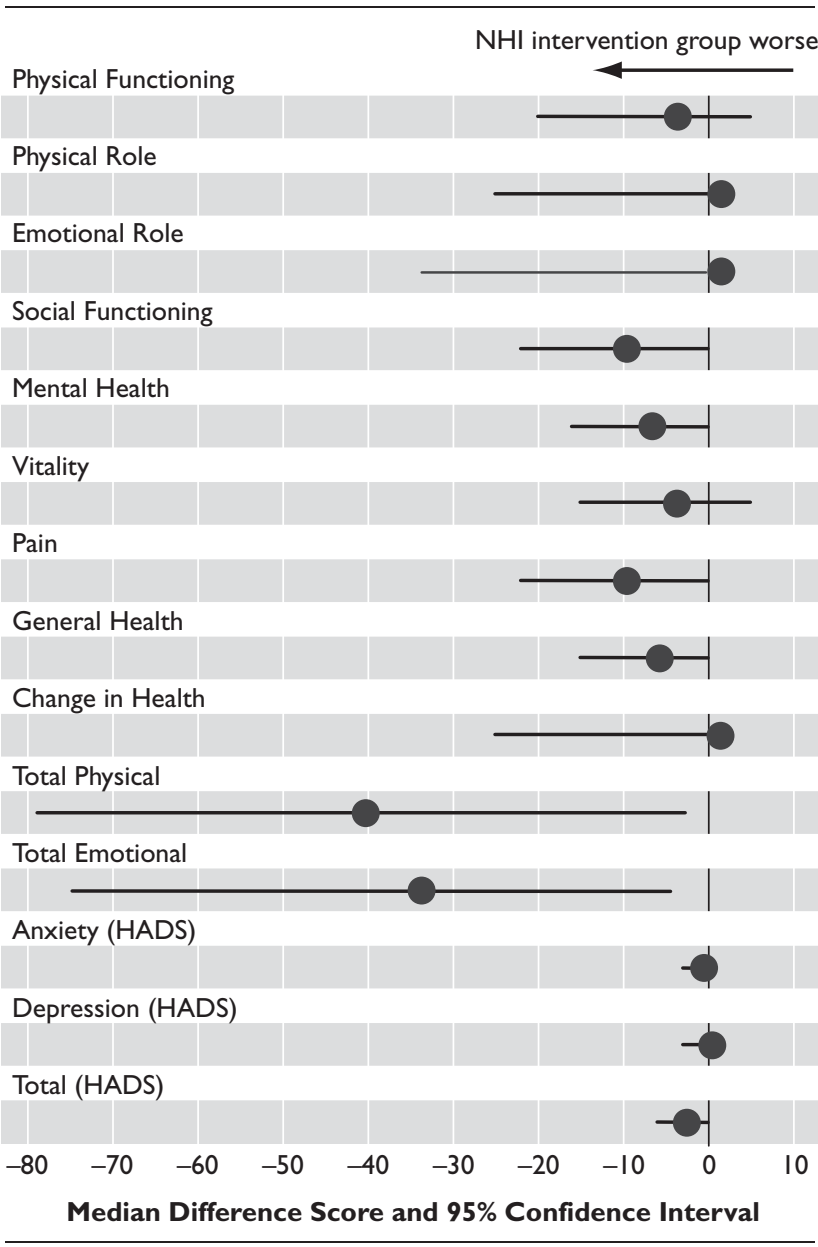


management intervention group, could also explain the lack of favorable outcomes for the intervention. Since our study was not designed to measure the complexity of care or the severity of illness in both groups, we used "quality of life on admission" as a proxy for complexity or severity $^{14,20}$ and controlled in the analysis for baseline differences in quality of life and psychological functioning. In doing so, we found no indications that differences in complexity or severity explain the results.

Differential work-up could also explain why the casemanagement intervention had no positive effect: patients in the case-management intervention group were more "looked-after," received more medical care, and were referred more frequently (Table 3). It has previously been suggested that case-management intervention programs lead to better care, but not to fewer emergency readmissions and less care-utilization. ${ }^{21}$

If the case-management intervention provides better care, we would have expected favorable outcomes for patients in the case-management intervention group in the domains of quality of life and psychological functioning. However, this was not the case, which made us speculate that the patients in the case-management intervention group could be more aware of their impaired functioning, since the case manager discussed these aspects extensively as part of the intervention, in contrast to patients in the control group, with whom this was not discussed. ${ }^{22}$ We did not measure patient satisfaction formally, but, in individual contacts, the patients reported that they were highly satisfied with the case-management intervention. Similar studies have found that patients were significantly more satisfied with case-management intervention, as compared with standard care..$^{21,23-25}$ If readmissions, care-utilization, and quality-of-life aspects are not positively influenced by case-management intervention, the question remains, in view of the amount of care-utilization and effort involved, whether the case-management intervention should be applied only on the basis of patient satisfaction.

A clinical explanation for the unfavorable outcomes of the case-management intervention is that we doubt whether the intervention was applied to its full extent, since the cooperation with primary care was not optimal. At the start of the study, it was apparent that many general practitioners had reservations about the aims of the study, and very few were of the opinion that case-management would be supportive for their practice. Communication lines were long, and it was difficult to formalize these. The number of healthcare workers varied per patient. There was an endless number of combinations of patients versus healthcare workers. It was difficult to inform all persons in question beforehand, and there was a lack of information about each others' knowledge and working domain. In one patient, the general-practitioner, the district nurse, the psychologist, and the case manager of this research were all of the opinion that they were supposed to be the case-manager for this patient.

In other words: the case-management intervention was based in a secondary-care facility, and the case-manager implemented the case-management intervention in a primary-care setting without full commitment of the general-practitioners and other primary healthcare workers. This is contrary to what was intended: to integrate this period of preventive care into primary care. In the Netherlands, the general-practitioner plays a central role in primary healthcare (whereas, for instance, in the United States, the nurse-practitioner plays an important role in outpatient health centers). The problems we experienced would be the same if we performed this study elsewhere than in the Netherlands. We expect the coordination problems experienced between the various disciplines to be similar in other countries, regardless of the differences in healthcare systems.

In summary, disease-management is effective, but may not be sufficient for complex patients. Case-management based on the nurse-led, home-based case-management intervention was not effective in terms of reducing emergency readmissions, reducing care-utilization, or in improving quality of life and psychological functioning. This finding suggests that these outcomes cannot be expected as a result of improved care for patients who have recently been discharged. Case-management intervention should be limited to complex patients and firmly embedded in the primary-care system before it can be effective. The intervention was sufficiently intensive for the post-discharge period, but it might have been more effective if the casemanager had visited the patient before discharge. Casemanagement should start in the hospital, so that the casemanager can formulate a care plan and discuss this with the primary-care team beforehand, with attention to the transition from hospital to home. Primary healthcare needs can be organized in a timely fashion, whereby the generalpractitioner and other primary healthcare workers agree on what to do the moment the patient is discharged. So, in our opinion, the case-manager should be based in primary care (located at a general-practitioner practice or an outpatient clinic), and have a formalized relationship with the hospital.

Nurse-led, home-based case-management intervention 


\section{Post-Discharge Case Management}

may result in higher care-utilization, but, at the same time, in improved care and a desirable shift of care toward homecare services and away from non-independent-living accommodations.
The Dutch Health Insurance Council supported this study with a research grant (00251; "Prevention of Re-Admissions by Means of Regional Care Coordination”) to Dr. P. de Jonge.

\begin{tabular}{|c|c|c|c|}
\hline & Control Group $(\mathrm{N}=17)$ & Intervention Group $(\mathrm{N}=9)$ & $\mathbf{p}$ \\
\hline Age, years, mean (SD) & $61.00(18.99)$ & $60.44(18.91)$ & 0.75 \\
\hline \multicolumn{4}{|l|}{ Gender, N (\%) } \\
\hline Male & $11(64.7)$ & $6(66.7)$ & \multirow[t]{2}{*}{0.92} \\
\hline Female & $6(35.3)$ & $3(33.3)$ & \\
\hline \multicolumn{4}{|l|}{ Marital status, N (\%) } \\
\hline Married/living together & $9(52.9)$ & $5(55.6)$ & \multirow[t]{2}{*}{0.90} \\
\hline Living alone & $8(47.1)$ & $4(44.4)$ & \\
\hline \multicolumn{4}{|l|}{ Work status, $\mathrm{N}(\%)$} \\
\hline Not working & $16(94.1)$ & $7(77.8)$ & \multirow[t]{3}{*}{0.22} \\
\hline Working & $1(5.2)$ & $2(22.2)$ & \\
\hline Length of stay in hospital, days, mean (SD) & $17.71(21.75)$ & $7.33(2.23)$ & \\
\hline \multicolumn{3}{|l|}{ Specialty, N (\%) } & \multirow[t]{5}{*}{0.15} \\
\hline Internal medicine & $9(52.9)$ & $5(55.6)$ & \\
\hline Gastroenterology & $1(5.9)$ & - & \\
\hline Pulmonology & $1(5.9)$ & $2(22.2)$ & \\
\hline Cardiology & $6(35.3)$ & $2(22.2)$ & \\
\hline Number of admissions in previous 5 years, mean (SD) & $2.59(3.20)$ & $1.67(1.00)$ & 0.56 \\
\hline INTERMED score, mean (SD) & - & - & \\
\hline \multicolumn{4}{|l|}{ Mini-Mental State Exam, N (\%) } \\
\hline$<21$ & 0 & 0 & \\
\hline$\geq 21$ & $17(100)$ & $9(100)$ & \\
\hline
\end{tabular}

\begin{tabular}{|c|c|c|c|}
\hline & Control Group $(\mathrm{N}=38)$ & Intervention Group $(\mathrm{N}=\mathbf{2 3})$ & $\mathbf{p}$ \\
\hline Age, years, mean (SD) & $69.13(16.66)$ & $66.0(20.15)$ & 0.80 \\
\hline \multicolumn{4}{|l|}{ Gender, $N(\%)$} \\
\hline Male & $21(55.3)$ & $10(43.5)$ & \multirow[t]{2}{*}{0.38} \\
\hline Female & $17(44.7)$ & $13(56.5)$ & \\
\hline \multicolumn{4}{|l|}{ Marital status, N (\%) } \\
\hline Married, living together & $16(42.1)$ & $10(43.5)$ & \multirow[t]{2}{*}{0.92} \\
\hline Living alone & $22(57.9)$ & $13(56.5)$ & \\
\hline \multicolumn{4}{|l|}{ Work status, $\mathrm{N}(\%)$} \\
\hline Not working & $35(92.1)$ & $18(78.3)$ & \multirow[t]{2}{*}{0.12} \\
\hline Working & $3(7.9)$ & $5(21.7)$ & \\
\hline Length of stay in hospital, days, mean (SD) & $12.18(10.43)$ & $10.3(6.70)$ & 0.63 \\
\hline \multicolumn{4}{|l|}{ Specialty, N (\%) } \\
\hline Internal medicine & $25(65.8)$ & $16(69.6)$ & \\
\hline Gastroenterology & $3(7.9)$ & $2(8.7)$ & \\
\hline Pulmonology & $3(7.9)$ & $2(8.7)$ & \\
\hline Cardiology & $7(18.4)$ & $3(13.0)$ & \\
\hline Number of admissions in previous 5 years, mean (SD) & $1.89(1.16)$ & $2.65(1.77)$ & 0.08 \\
\hline INTERMED score, mean (SD) & - & - & \\
\hline \multicolumn{4}{|l|}{ Mini-Mental State Exam score, N $(\%)^{\mathrm{b}}$} \\
\hline$<21$ & $1(2.6)^{\mathrm{a}}$ & $-^{\mathrm{b}}$ & \\
\hline$\geq 21$ & $35(92.1)^{\mathrm{a}}$ & $22(9.6)^{\mathrm{b}}$ & 0.81 \\
\hline $\begin{array}{l}\text { Drop-out patients were those who withdrew consent, } \\
\text { a } 2 \text { cases missing. } \\
{ }^{b} 1 \text { case missing. }\end{array}$ & were lost to follow-up. & & \\
\hline
\end{tabular}




\section{References}

1. Aubert RE, Herman WH, Waters J, et al: Nurse case-management to improve glycemic control in diabetic patients in a health maintenance organization: a randomized, controlled trial. Ann Intern Med 1998; 129:605-612

2. Harris LE, Luft FC, Rudy DW, et al: Effects of multidisciplinary case-management in patients with chronic renal insufficiency. Am J Med 1998; 105:464-471

3. Stewart S, Marley JE, Horowitz JD: Effects of a multidisciplinary, home-based intervention on unplanned readmissions and survival among patients with chronic congestive heart failure: a randomised, controlled study. Lancet 1999; 354:1077-1083

4. Stuck AE, Siu AL, Wieland GD, et al: Comprehensive geriatric assessment: a meta-analysis of controlled trials. Lancet 1993; 342:1032-106

5. West JA, Miller NH, Parker KM, et al: A comprehensive management system for heart failure improves clinical outcomes and reduces medical resource utilization. Am J Cardiol 1997; 79:58-63

6. Leveille SG, Wagner EH, Davis C, et al: Preventing disability and managing chronic illness in frail older adults: a randomized trial of a community-based partnership with primary care. J Am Geriatr Soc 1998; 46:1191-1198

7. Naylor MD, Brooten D, Campbell R, et al: Comprehensive discharge planning and home follow-up of hospitalized elders: a randomized clinical trial. JAMA 1999; 281:613-620

8. Rich MW, Beckham V, Wittenberg C, et al: A multidisciplinary intervention to prevent the readmission of elderly patients with congestive heart failure. N Engl J Med 1995; 333:1190-115

9. Curley C, McEachern JE, Speroff T: A firm trial of interdisciplinary rounds on the inpatient medical wards: an intervention designed using continuous quality improvement. Med Care 1998; 36(supp18):AS4-AS12

10. de Jonge P, Huyse FJ, Stiefel FC, et al: INTERMED: a clinical instrument for biopsychosocial assessment. Psychosomatics 2001; 42:106-109

11. de Jonge P, Latour C, Huyse FJ: Interrater reliability of the INTERMED in a heterogeneous somatic population. J Psychosom Res 2002; 52:25-27

12. Stiefel FC, de Jonge P, Huyse FJ, et al: "INTERMED": a method to assess health service needs, II: results on its validity and clinical use. Gen Hosp Psychiatry 1999; 21:49-56

13. de Jonge $\mathrm{P}$, Latour $\mathrm{CH}$, Huyse FJ: Implementing psychiatric in- terventions on a medical ward: effects on patients' quality of life and length of hospital stay. Psychosom Med 2003; 65:997-1002

14. de Jonge P, Bauer I, Huyse FJ, et al: Medical inpatients at risk of extended hospital stay and poor discharge health status: detection with COMPRI and INTERMED. Psychosom Med 2003; 65:534541

15. Folstein MF, Folstein SE, McHugh PR: "Mini-Mental State:" a practical method for grading the cognitive state of patients for the clinician. J Psychiatr Res 1975; 12:189-198

16. Katz S: Assessing self-maintenance: activities of daily living, mobility, and instrumental activities of daily living. J Am Geriatr Soc 1983; 31:721-727

17. McHorney CA, Ware JE Jr, Raczek AE: The MOS 36-Item ShortForm Health Survey (SF-36), II: psychometric and clinical tests of validity in measuring physical and mental health constructs. Med Care 1993; 31:247-263

18. Ware JE Jr: SF-36 Health Survey update. Spine 2000; 25:3130319

19. Zigmond AS, Snaith RP: The Hospital Anxiety and Depression Scale. Acta Psychiatr Scand 1983; 67:361-370

20. Vedsted P, Fink P, Sorensen HT, et al: Physical, mental, and social factors associated with frequent attendance in Danish general practice: a population-based, cross-sectional study. Soc Sci Med 2004; 59:813-823

21. Weinberger M, Oddone EZ, Henderson WG: Does increased access to primary care reduce hospital readmissions? Veterans Affairs Cooperative Study Group on Primary Care and Hospital Readmission. N Engl J Med 1996; 334:1441-147

22. Frasure-Smith N, Lesperance F, Prince RH, et al: Randomised trial of home-based psychosocial nursing intervention for patients recovering from myocardial infarction. Lancet 1997; 350:473-479

23. Laramee AS, Levinsky SK, Sargent J, et al: Case-management in a heterogeneous congestive heart failure population: a randomized controlled trial. Arch Intern Med 2003; 163:809-817

24. Litaker D, Mion L, Planavsky L, et al: Physician-nurse-practitioner teams in chronic disease management: the impact on costs, clinical effectiveness, and patients' perception of care. Journal of Interprofessional Care 2003; 17:223-237

25. Hermiz O, Comino E, Marks G, et al: Randomised, controlled trial of home-based care of patients with chronic obstructive pulmonary disease. BMJ 2002; 325:938 